\title{
Inverse Hyperbolic Problems and Optical Black Holes
}

\author{
G. Eskin \\ Department of Mathematics, UCLA, Los Angeles, CA 90095-1555, USA. E-mail: eskin@math.ucla.edu
}

Received: 3 March 2009 / Accepted: 16 March 2010

Published online: 23 May 2010 - (C) The Author(s) 2010. This article is published with open access at Springerlink.com

To the memory of Leonid Romanovich Volevich

Abstract: In this paper we state a uniqueness theorem for the inverse hyperbolic problem in the case of a finite time interval. We apply this theorem to the inverse problem for the equation of the propagation of light in a moving medium (the Gordon equation). Then we study the existence of black and white holes for the general second order hyperbolic equation and for the Gordon equation and we discuss the impact of this phenomenon on the inverse problems.

\section{The Introduction and the Statement of the Main Theorem}

We start with the formulation of the inverse problem. Let $\Omega$ be a smooth bounded domain in $\mathbf{R}^{n}$. Consider a second order hyperbolic equation in a cylinder $\Omega \times(-\infty,+\infty)$ :

$$
\sum_{j, k=0}^{n} \frac{1}{\sqrt{(-1)^{n} g(x)}} \frac{\partial}{\partial x_{j}}\left(\sqrt{(-1)^{n} g(x)} g^{j k}(x) \frac{\partial u\left(x_{0}, x\right)}{\partial x_{k}}\right)=0,
$$

where $x=\left(x_{1}, \ldots, x_{n}\right) \in \Omega, x_{0} \in \mathbf{R}$ is the time variable,

$g(x)=\left(\operatorname{det}\left[g^{j k}(x)\right]_{j, k=0}^{n}\right)^{-1}$. We assume that $g^{j k}(x)=g^{k j}(x)$ are real-valued smooth functions in $C^{\infty}(\bar{\Omega})$ independent of $x_{0}$.

The hyperbolicity of (1.1) means that the quadratic form $\sum_{j, k=0}^{n} g^{j k}(x) \xi_{j} \xi_{k}$ has one positive and $n$ negative eigenvalues, $\forall x \in \bar{\Omega}$. We assume in addition that

$$
g^{00}(x)>0, \quad x \in \bar{\Omega},
$$

i.e. $(1,0, \ldots, 0)$ is not a characteristic direction, and that

$$
\sum_{j, k=1}^{n} g^{j k}(x) \xi_{j} \xi_{k}<0 \quad \text { for } \forall\left(\xi_{1}, \ldots, \xi_{n}\right) \neq(0, \ldots, 0), \quad \forall x \in \bar{\Omega},
$$


i.e. the quadratic form (1.3) is negative definite. Note that (1.3) is equivalent to the condition that

$$
\text { any direction }(0, \xi) \text { is not characteristic for } \forall x \in \bar{\Omega} \text {. }
$$

We shall give later another equivalent characterization of the condition (1.3).

We shall study the initial-boundary value problem for Eq. (1.1) in $\Omega \times \mathbf{R}$,

$$
\begin{aligned}
& u\left(x_{0}, x\right)=0 \text { for } x_{0}<<0, \quad x \in \Omega, \\
& \left.u\right|_{\partial \Omega \times \mathbf{R}}=f,
\end{aligned}
$$

where $f\left(x_{0}, x^{\prime}\right)$ has compact support in $\partial \Omega \times \mathbf{R}$.

Consider the Dirichlet-to-Neumann (DN) operator

$$
\Lambda f=\left.\sum_{j, k=0}^{n} g^{j k}(x) \frac{\partial u}{\partial x_{j}} v_{k}(x)\left(\sum_{p, r=1}^{n} g^{p r}(x) v_{p} v_{r}\right)^{-\frac{1}{2}}\right|_{\partial \Omega \times \mathbf{R}},
$$

where $v_{0}=0,\left(v_{1}, \ldots, v_{n}\right)$ is the unit outward normal to $\partial \Omega \subset \mathbf{R}^{n}$ with respect to the Euclidean metric, $u\left(x_{0}, x\right)$ is the solution of (1.1), (1.5), (1.6).

Let $\Gamma_{0} \subset \partial \Omega$ be an open subset of $\partial \Omega$. We shall say that the DN operator $\Lambda$ is given on $\Gamma_{0} \times\left(0, T_{0}\right)$ if we know $\left.\Lambda f\right|_{\Gamma_{0} \times\left(0, T_{0}\right)}$ for any smooth $f$ with support in $\bar{\Gamma}_{0} \times\left[0, T_{0}\right]$.

Let $X$ be a closed compact subset of $\partial \Omega \times(-\infty,+\infty)$. For each distribution $f$ on $\partial \Omega \times(-\infty,+\infty)$, supp $f \subset X$, denote by $D_{+}(f)$ the support in $\bar{\Omega} \times(-\infty,+\infty)$ of the solution of the initial-boundary value problem for (1.1) with $u=0$ for $x_{0}<<0, x \in$ $\Omega,\left.u\right|_{\partial \Omega \times(-\infty,+\infty)}=f$.

We define the forward domain of influence of $X$ as the closure of the set $\cup_{\text {supp } f \subset X} D_{+}(f)$, where the union is taken over all $f$ on $\partial \Omega \times(-\infty,+\infty)$ with supports in $X$.

Analogously, let $D_{-}(f)$ be the support in $\bar{\Omega} \times(-\infty, \infty)$ of the solution of the initial boundary value problem for (1.1) with $u=0$ for $x_{0}>0, x \in \Omega,\left.u\right|_{\partial \Omega \times(-\infty,+\infty)}=f$. Then $D_{-}(X)$ is the closure of the union of all $D_{-}(f)$ with supp $f \subset X$.

We shall give a geometrical description of the set $D_{+}(X)$.

Let $\left[g_{j k}(x)\right]_{j, k=0}^{n}=\left(\left[g^{j k}\right]_{j, k=0}^{n}\right)^{-1}$, i.e. $\left[g_{j k}(x)\right]_{j, k=0}^{n}$ is the pseudo-Riemannian metric tensor. We say that $\gamma(s)=\left(x_{0}(s), x_{1}(s), \ldots, x_{n}(s)\right), s \in\left[0, s_{0}\right]$ is a forward time-like path (ray) if $\gamma(s)$ is continuous and piece-wise smooth and each smooth segment $\gamma^{(i)}(s)$ of $\gamma(s)$ satisfies

$$
\sum_{j, k=0}^{n} g_{j k}(x(s)) \frac{d x_{j}}{d s} \frac{d x_{k}}{d s}>0, \quad s_{i} \leq s \leq s_{i+1},
$$

$\frac{d x_{0}}{d s}>0$. Then $D_{+}(X)$ is the closure in $\bar{\Omega} \times(-\infty,+\infty)$ of all time-like rays starting on $X$. Note that the vertical ray $x_{0}=s, x=x^{(0)}, s \geq 0$, is time-like iff $g_{00}\left(x^{(0)}\right)>0$.

Proposition 1.1. Condition (1.3) holds iff $g_{00}(x)>0$, i.e. the ray $x_{0}=s, x \in \bar{\Omega}, s \geq 0$, is time-like. 
Proof. We have

$$
g_{00}(x)=\frac{\operatorname{det}\left[g^{j k}\right]_{j, k=1}^{n}}{g^{-1}(x)}
$$

Note that

$$
\begin{aligned}
\sum_{j, k=0}^{n} g^{j k}(x) \xi_{j} \xi_{k}= & \left(\sqrt{g^{00}(x)} \xi_{0}+\sum_{j=1}^{n} \frac{g^{0 j}(x)}{\sqrt{g_{00}(x)}} \xi_{j}\right)^{2} \\
& -\left(\sum_{j=1}^{n} \frac{g^{0 j}(x)}{\sqrt{g_{00}(x)}} \xi_{j}\right)^{2}+\sum_{j, k=1}^{n} g^{j k}(x) \xi_{j} \xi_{k} .
\end{aligned}
$$

Since the quadratic form $\sum_{j, k=0}^{n} g^{j k} \xi_{j} \xi_{k}$ has the signature $(+1,-1, \ldots,-1)$ the quadratic form

$$
-\left(\sum_{j=1}^{n} \frac{g^{j 0}(x)}{\sqrt{g_{00}(x)}} \xi_{j}\right)^{2}+\sum_{j, k=1}^{n} g^{j k}(x) \xi_{j} \xi_{k}
$$

is negative definite. Therefore $\sum_{j, k=1}^{n} g^{j k}(x) \xi_{j} \xi_{k}$ either has only negative eigenvalues, or it has one zero eigenvalue, or it has one positive eigenvalue. In the first case $\operatorname{sgn}\left(\operatorname{det}\left[g^{j k}\right]_{j, k=1}^{n}\right)=(-1)^{n}$, in the second case $\operatorname{det}\left[g^{j k}(x)\right]_{j, k=1}^{n}=0$, and in the third case $\operatorname{sgn}\left(\operatorname{det}\left[g^{j k}\right]_{j, k=1}^{n}\right)=(-1)^{n-1}$. Since $\operatorname{sgn} g(x)=(-1)^{n}$ we get that $g_{00}(x)>0$ iff condition (1.3) holds.

Let $T_{+}$be the smallest number such that $D_{+}\left(\bar{\Gamma}_{0} \times\left\{x_{0}=0\right\}\right) \supset \bar{\Omega} \times\left\{x_{0}=T_{+}\right\}$. Analogously let $T_{-}$be the smallest number such that $D_{-}\left(\bar{\Gamma}_{0} \times\left\{x_{0}=T_{-}\right\}\right) \supset \bar{\Omega} \times\left\{x_{0}=0\right\}$.

We shall require that

$$
T_{0}>T_{+}+T_{-} .
$$

Consider the following change of variables in $\bar{\Omega} \times(-\infty,+\infty)$ :

$$
\begin{aligned}
y_{0} & =x_{0}+a(x), \\
y & =\varphi(x),
\end{aligned}
$$

where $y=\varphi(x)$ is a diffeomorphism of $\bar{\Omega}$ onto $\overline{\tilde{\Omega}}, a(x) \in C^{\infty}(\bar{\Omega})$,

$$
\begin{aligned}
& \varphi(x)=I \text { on } \bar{\Gamma}_{0} \subset \partial \Omega, \\
& a(x)=0 \text { on } \bar{\Gamma}_{0} .
\end{aligned}
$$

Note that if $\tilde{u}\left(y_{0}, y\right)=u\left(x_{0}, x\right)$, where $\left(y_{0}, y\right)$ and $\left(x_{0}, x\right)$ are related by (1.10), (1.11), then $\tilde{u}\left(y_{0}, y\right)$ satisfies an equation of the form

$$
\sum_{j, k=0}^{n} \frac{1}{\sqrt{(-1)^{n} \tilde{g}(y)}} \frac{\partial}{\partial y_{j}}\left(\sqrt{(-1)^{n} \tilde{g}(y)} \tilde{g}^{j k}(y) \frac{\partial \tilde{u}\left(y_{0}, y\right)}{\partial y_{k}}\right)=0
$$


in $\tilde{\Omega} \times(-\infty,+\infty)$ with initial and boundary conditions

$$
\begin{aligned}
\tilde{u}\left(y_{0}, y\right) & =0 \text { for } y_{0}<<0, \quad y \in \tilde{\Omega}, \\
\left.\tilde{u}\right|_{\partial \tilde{\Omega} \times \mathbf{R}} & =f\left(y_{0}, y^{\prime}\right),
\end{aligned}
$$

where $f\left(x_{0}, x^{\prime}\right)=f\left(y_{0}, y^{\prime}\right)$ on $\Gamma_{0} \times(-\infty,+\infty)$.

Let $\tilde{\Lambda}$ be the DN operator on $\Gamma_{0} \times(-\infty,+\infty)$ corresponding to (1.12), (1.13), (1.14).

The following theorem holds (cf. [E1], Theorem 2.3)

Theorem 1.1. Let (1.1), (1.5), (1.6) and (1.12), (1.13), (1.14) be two hyperbolic initial-boundary value problems in $\Omega \times \mathbf{R}$ and $\tilde{\Omega} \times \mathbf{R}$, respectively, and $\partial \Omega \cap \partial \tilde{\Omega} \supset \Gamma_{0}$. Suppose conditions (1.2) and (1.3) are satisfied for (1.1) and (1.12). Suppose supp $f \subset$ $\bar{\Gamma}_{0} \times\left[0, T_{0}\right]$ in (1.6) and (1.14). If the DN operators $\Lambda$ and $\tilde{\Lambda}$ are equal on $\Gamma_{0} \times\left(0, T_{0}\right)$ and if $T_{0}>T_{+}+T_{-}$(cf. (1.9)) then there exists a change of variables (1.10), (1.11) such that

$$
\left[\tilde{g}^{j k}(y)\right]_{j, k=0}^{n}=J(x)\left[g^{j k}(x)\right]_{j, k=0}^{n} J^{T}(x),
$$

where $y_{0}=x_{0}+a(x), y=\varphi(x)$,

$$
J(x)=\left[\begin{array}{ll}
1 & a_{x}(x) \\
0 & \frac{\mathcal{D} \varphi(x)}{\mathcal{D} x}
\end{array}\right]
$$

is the Jacobi matrix of (1.10).

Note that condition (1.9) is required for (1.1) only. The condition (1.15) is equivalent to

$$
\left[\tilde{g}^{j k}(y)\right]^{-1}=\left(J^{T}(x)\right)^{-1}\left[g^{j k}(x)\right]^{-1} J^{-1}(x),
$$

or

$$
\left[g_{j k}(x)\right]_{j, k=0}^{n}=J^{T}(x)\left[\tilde{g}_{j k}(y)\right]_{j, k=0}^{n} J(x) .
$$

The equality (1.17) can be rewritten as the equality of differential forms

$$
\sum_{j, k=0}^{n} g_{j k}(x) d x_{j} d x_{k}=\sum_{j, k=1}^{n} \tilde{g}_{j k}(y) d y_{j} d y_{k} .
$$

Theorem 1.1 is a refinement of Theorem 2.3 in [E1] when the time-interval $(-\infty,+\infty)$ is replaced by $\left(0, T_{0}\right)$, and it does not require any changes in the proof of Theorem 2.3 in [E1] (see also [E2]).

Now we shall describe the content of the rest of the paper.

In Sect. 2 we apply Theorem 1.1 to the inverse problem for the equation of the propagation of light in a moving medium (the Gordon equation).

In Sects. 3 and 4 we find the conditions for the existence of the black or white holes for the equation of the form (1.1). Note that the black or white holes in $\S 4$ are stable, i.e. they change only slightly when the metric is slightly changed.

The phenomenon of black holes for the wave equation (1.1) attracted the attention of physicists (see, for example, the survey of M.Visser [V], and the book "Artificial black holes" by M.Novello, M.Visser and G.Volovik [NVV]). The physicists were interested in studying these black holes (they are called either artificial black holes, or acoustic 
black holes, or optical black holes) as a "laboratory" for the black holes arising in the Einstein equations of general relativity.

The relation between the inverse problems in $\S \S 1-2$ and the black holes in $\S \S 3-4$ is the following: Black or white holes may appear only when the condition (1.3) in the uniqueness Theorem 1.1 is violated. The existence of black or white holes leads to the non-uniqueness of the solution of the inverse problem.

\section{The Inverse Problem for the Gordon Equation}

Consider the equation of the propagation of light in a moving medium. Let $w(x)=$ $\left(w_{1}(x), w_{2}(x), w_{3}(x)\right)$ be the velocity of flow and let $\left(v^{(0)}, v^{(1)}, v^{(2)}, v^{(3)}\right)$ be the corresponding four-velocity vector

$$
v^{(0)}=\left(1-\frac{|w|^{2}}{c^{2}}\right)^{-\frac{1}{2}}, \quad v^{(j)}=\left(1-\frac{|w|^{2}}{c^{2}}\right)^{-\frac{1}{2}} \frac{w_{j}(x)}{c}, \quad 1 \leq j \leq 3,
$$

where $c$ is the speed of light in the vacuum (cf. [G,NVV,LP]). The equation for the propagation of light was found in 1923 by Gordon (cf. [G]) and it has the form (1.1) when $g^{j k}(x), 0 \leq j, k \leq n, n=3$, are

$$
g^{j k}(x)=\eta^{j k}+\left(n^{2}(x)-1\right) v^{j}(x) v^{k}(x),
$$

$\left[\eta_{j k}\right]=\left[\eta^{j k}\right]^{-1}$ is the Lorenz metric tensor: $\eta^{j k}=0$ when $j \neq k, \eta^{00}=1, \eta^{j j}=-1$, $1 \leq j \leq 3, n(x)=\sqrt{\varepsilon(x) \mu(x)}$ is the refraction index. Note that $\left[g_{j k}(x)\right]=\left[g^{j k}\right]^{-1}$ has the form (cf. [LP]):

$$
g_{j k}(x)=\eta_{j k}+\left(n^{-2}(x)-1\right) v_{j} v_{k}, \quad 0 \leq j, k \leq n,
$$

where $v_{0}=v^{0}, v_{j}=-v^{j}, 1 \leq j \leq n$.

The metric (2.2) is called the Gordon metric and the corresponding equation of the form (1.1) is called the Gordon equation. We shall consider the Gordon equation in $\Omega \times(-\infty,+\infty)$, where $\Omega$ has the form $\Omega=\Omega_{0} \backslash \cup_{j=1}^{m} \bar{\Omega}_{j}, \Omega_{0}$ is diffeomorphic to a ball, $\Omega_{j}$ are smooth domains, $\bar{\Omega}_{j} \cap \bar{\Omega}_{k}=\emptyset$ when $j \neq k$ and $\cup_{j=1}^{m} \bar{\Omega}_{j} \subset \Omega$. Domains $\Omega_{j}$ are called obstacles.

We shall study the following initial-boundary value problem for the Gordon equation:

$$
\begin{aligned}
& u\left(x_{0}, x\right)=0 \text { for } x_{0}<<0, x \in \Omega, \\
& \left.u\left(x_{0}, x\right)\right|_{\partial \Omega_{j} \times \mathbf{R}}=0, \quad 1 \leq j \leq m, \\
& \left.u\left(x_{0}, x\right)\right|_{\partial \Omega_{0} \times \mathbf{R}}=f\left(x_{0}, x\right),
\end{aligned}
$$

i.e. $\Gamma_{0}=\partial \Omega_{0}$, where $\Gamma_{0}$ is the same as in Theorem 1.1. One can consider also the case when on some or all obstacles $\Omega_{j}$ the zero Dirichlet boundary condition is replaced by the zero Neumann boundary condition.

Note that the condition (1.2) always holds for the Gordon equation

$$
g^{00}=1+\left(n^{2}-1\right)\left(v^{0}\right)^{2}>0 .
$$

The condition (1.3) holds iff

$$
|w(x)|^{2}<\frac{c^{2}}{n^{2}(x)}
$$


To prove (2.7) note that the principal symbol of the Gordon equation is

$$
\xi_{0}^{2}-|\xi|^{2}+\left(n^{2}-1\right)\left(\sum_{j=0}^{n} v^{j} \xi_{j}\right)^{2}
$$

Therefore the quadratic form

$$
-|\xi|^{2}+\left(n^{2}-1\right)\left(\sum_{j=1}^{n} v^{j} \xi_{j}\right)^{2}
$$

is negative definite iff $\left(n^{2}-1\right)|v|^{2}<1$. Since $v^{0}=\left(1-\frac{|w|^{2}}{c^{2}}\right)^{-\frac{1}{2}}, v^{j}=\left(1-\frac{|w|^{2}}{c^{2}}\right)^{-\frac{1}{2}}$ $\frac{w_{j}}{c}, 1 \leq j \leq n$, we get (2.7).

We shall study the inverse problem for the Gordon equation. The case of slowly moving medium (cf. [LP]) was considered in [E1]. The Dirichlet-to-Neumann operator corresponding to the Gordon equation has the form (cf. (1.7)):

$$
\Lambda f=\left.\sum_{j, k=0}^{n}\left[\eta^{j k}+\left(n^{2}(x)-1\right) v^{j} v^{k}\right] \frac{\partial u}{\partial x_{j}} v_{k}\left|-1+\left(n^{2}-1\right)\left(\sum_{r=1}^{n} v^{r} v_{r}\right)^{2}\right|^{-\frac{1}{2}}\right|_{\partial \Omega_{0} \times \mathbf{R}},
$$

where $v(x)=\left(v_{1}, \ldots, v_{n}\right)$ is the unit outward normal vector to $\partial \Omega_{0}, v_{0}=0, u$ $\left.\right|_{\partial} \Omega_{0} \times \mathbf{R}=f$.

We shall impose some restriction on the flow $w(x)=\left(w_{1}(x), \ldots, w_{n}(x)\right)$.

Let $x=x(s), s \in\left[0, s_{0}\right]$ be a trajectory of the flow, i.e. $\frac{d x}{d s}=w(x(s)), w(x(s)) \neq$ $0, s \in\left[0, s_{0}\right]$. Consider the trajectories that either start and end on $\partial \Omega_{0}$ or are closed curves in $\Omega$. Our assumption is that

The union of all such trajectories is a dense set in $\Omega$.

Theorem 2.1. Let $\left[g_{j k}(x)\right]_{j, k=0}^{n}$ and $\left[\tilde{g}_{j k}(y)\right]_{j, k=0}^{n}$ be two Gordon metrics in domains $\Omega$ and $\tilde{\Omega}$, respectively, and $\partial \Omega_{0}=\partial \tilde{\Omega}_{0}$. Consider two initial-boundary value problems (1.1), (1.5), (1.6) and (1.12), (1.13), (1.14) corresponding to the metrics $\left[g_{j k}(x)\right]_{j, k=0}^{n}$ and $\left[\tilde{g}_{j k}(y)\right]_{j, k=0}^{n}$, respectively. Assume that the condition (2.7) holds for both metrics and assume that the refraction coefficients $n^{2}(x)$ and $\tilde{n}^{2}(y)$ are constant. Assume also that the flow $w(x)$ satisfies the condition (2.9). If the DN operators $\Lambda$ and $\tilde{\Lambda}$ corresponding to $\left[g_{j k}(x)\right]_{j, k=0}^{n}$ and $\left[\tilde{g}_{j k}(y)\right]_{j, k=0}^{n}$ are equal on $\partial \Omega_{0} \times\left(0, T_{0}\right)$, where $T_{0}$ satisfies (1.9) for Eq. (1.1), then we have

$$
n^{2}=\tilde{n}^{2}, \quad \Omega=\tilde{\Omega},
$$

and the flows $w(x)$ and $\tilde{w}(x)$ are equal.

Proof. Analogously to Remark 2.2 in [E1] we can find the symbol of the DN operator in the "elliptic" region (cf. [E1]) and retrieve $\left.n^{2}(x)\right|_{\partial \Omega_{0}}$. Since $\Lambda=\tilde{\Lambda}$ on $\partial \Omega_{0} \times\left(0, T_{0}\right)$ we get that $\left.n^{2}(x)\right|_{\partial \Omega_{0}}=\left.\tilde{n}^{2}(x)\right|_{\partial \Omega_{0}}$. Therefore $n^{2}=\tilde{n}^{2}$ in $\bar{\Omega}_{0}$ since we assume that $n^{2}$ and $\tilde{n}^{2}$ do not depend on $x$. Applying Theorem 1.1 to the case of the Gordon equation we 
get that there exists a change of variables (1.10) such that $a(x)=0$ on $\partial \Omega_{0}, \varphi(x)=I$ on $\partial \Omega_{0}$ and (1.15), (1.18) hold where

$$
\begin{array}{ll}
g^{j k}(x)=\eta^{j k}+\left(n^{2}-1\right) v^{j}(x) v^{k}(x), & 0 \leq j, k \leq n, \\
\tilde{g}^{j k}(y)=\eta^{j k}+\left(n^{2}-1\right) \tilde{v}^{j}(y) \tilde{v}^{k}(y), & 0 \leq j, k \leq n .
\end{array}
$$

We have from (1.10)

$$
\begin{aligned}
& d y_{0}=d x_{0}+\sum_{k=1}^{n} a_{x_{k}}(x) d x_{k}, \\
& d y_{j}=\sum_{k=1}^{n} \varphi_{j x_{k}}(x) d x_{k}, \quad 1 \leq k \leq n .
\end{aligned}
$$

Substituting (2.11) into (1.18) and taking into account that $d x_{0}, d x_{1}, \ldots, d x_{n}$ are arbitrary we get

$$
g_{00}(x)=\tilde{g}_{00}(y)
$$

Therefore (cf. (2.2))

$$
1+\left(n^{-2}-1\right) v_{0}^{2}(x)=1+\left(\tilde{n}^{-2}-1\right) \tilde{v}_{0}^{2}(y) .
$$

Note that (1.15) is equivalent to

$$
\tilde{g}^{j k}(y)=\sum_{p, r=0}^{n} g^{p r}(x) \varphi_{j x_{p}} \varphi_{k x_{r}},
$$

where $\varphi_{0}=x_{0}+a(x)$. In particular,

$$
\tilde{g}^{00}(y)=g^{00}(x)+2 \sum_{p=1}^{n} g^{p 0}(x) a_{x_{p}}(x)+\sum_{p, r=1}^{n} g^{p r}(x) a_{x_{p}} a_{x_{r}}(x) .
$$

In the case of Gordon metrics we have

$$
\begin{aligned}
1+ & \left(\tilde{n}^{2}-1\right)\left(\tilde{v}^{0}(y)\right)^{2} \\
= & 1+\left(n^{2}-1\right)\left(v^{0}(x)\right)^{2}+2 \sum_{p=1}^{n}\left(n^{2}-1\right) v^{0}(x) v^{p}(x) a_{x_{p}}(x) \\
& -\sum_{p=1}^{n}\left(a_{x_{x p}}(x)\right)^{2}+\sum_{p, r=1}^{n}\left(n^{2}-1\right) v^{p}(x) v^{r}(x) a_{x_{p}}(x) a_{x_{r}}(x) .
\end{aligned}
$$

Denote

$$
|\nabla a(x)|^{2}=\sum_{p=1}^{n} a_{x_{p}}^{2}(x), \quad a_{0}(x)=\frac{1}{c} \sum_{p=1}^{n} w_{p}(x) a_{x_{p}}(x) .
$$

Since $n=\tilde{n}$ and since (2.12) implies that $v^{0}=\tilde{v}^{0}$ we get

$$
a_{0}^{2}+2 a_{0}(x)-\frac{|\nabla a|^{2}}{\left(v^{0}\right)^{2}\left(n^{2}-1\right)}=0 .
$$


It follows from (2.16) that either

$$
a_{0}=-1-\sqrt{1+\frac{|\nabla a|^{2}}{\left(v^{0}\right)^{2}\left(n^{2}-1\right)}}
$$

or

$$
a_{0}=-1+\sqrt{1+\frac{|\nabla a|^{2}}{\left(v^{0}\right)^{2}\left(n^{2}-1\right)}} .
$$

Let $x=x(s)$ be a trajectory of the flow, i.e.

$$
\frac{d x_{k}}{d s}=w_{k}(x(s)), \quad 1 \leq k \leq n, \quad 0 \leq s \leq 1 .
$$

Then

$$
\frac{d a(x(s))}{d s}=\sum_{k=1}^{n} a_{x_{k}}(x(s)) \frac{d x_{k}}{d s}=c a_{0}(x(s)),
$$

i.e. $c a_{0}(x(s))$ is the derivative of $a(x(s))$ along the trajectory of the flow.

Suppose $x=x(s), 0 \leq s \leq 1$, is a trajectory that starts and ends on $\partial \Omega_{0}$. Therefore $a(x(0))=a(x(1))=0$ since $a(x)=0$ on $\partial \Omega_{0}$. Then $a_{0}(x(s))$ can not satisfy (2.17) since $\frac{d a(x(s))}{d s}=c a_{0}(x(s))<0$ on $[0,1]$. Therefore $\frac{d a(x(s))}{d s}=c a_{0}(x(s))$ satisfies Eq. (2.18), i.e. $\frac{d a(x(s))}{d s} \geq 0$ on [0,1]. Since $a(x(0))=a(x(1))=0$ we must have $a(x(s))=0$ and $\nabla a(x(s))=0$ on $[0,1]$. In the case when $x=x(s), 0 \leq s \leq 1$, is a closed trajectory, i.e. $x(0)=x(1), a(x(s))$ can not again satisfy (2.17) since $\frac{d a(\bar{x}(s))}{d s}<0$ on $[0,1]$ and $a(x(s))$ satisfies (2.18) only when $\nabla a(x(s))=0$ and $a(x(s))=$ const on $[0,1]$.

Since the condition (2.9) holds we have $\nabla a(x)=0$ in $\Omega$, and since $\left.a\right|_{\partial \Omega_{0}}=0$, we get that $a=0$ in $\bar{\Omega}$.

Applying (2.13) for $k=0,1 \leq j \leq n$, and for $1 \leq j, k \leq n$ and taking into account that $a \equiv 0$ we get

$$
\begin{aligned}
\left(\tilde{n}^{2}-1\right) \tilde{v}^{0}(y) \tilde{v}^{j}(y)= & \left(n^{2}-1\right) \sum_{p=1}^{n} v^{0}(x) v^{p}(x) \varphi_{j x_{p}}(x), \\
\eta^{j k}+\left(\tilde{n}^{2}-1\right) \tilde{v}^{j}(y) \tilde{v}^{k}(y)= & -\sum_{p=1}^{n} \varphi_{j x_{p}} \varphi_{k x_{p}} \\
& +\sum_{p, r=1}^{n}\left(n^{2}-1\right) v^{p}(x) v^{r}(x) \varphi_{j x_{p}} \varphi_{k x_{r}}, \quad 1 \leq j, k \leq n .
\end{aligned}
$$

Using again that $\tilde{n}=n$ and $v^{0}=\tilde{v}^{0}$ we get from (2.19)

$$
\tilde{v}^{j}(y)=\sum_{p=1}^{n} v^{p}(x) \varphi_{j x_{p}}(x), \quad 1 \leq j \leq n, \tilde{v}^{0}(y)=v^{0}(x) .
$$


Substituting (2.21) into (2.20) we obtain

$$
\eta^{j k}=-\sum_{p=1}^{n} \varphi_{j x_{p}}(x) \varphi_{k x_{p}}(x), \quad 1 \leq j, k \leq n .
$$

In particular, we have

$$
\sum_{p=1}^{n} \varphi_{j x_{p}}^{2}(x)=1,\left.\quad \varphi_{j}\right|_{\partial \Omega_{0}}=x_{j}, \quad 1 \leq j \leq n .
$$

The Cauchy problem (2.23) has a unique solution in $\bar{\Omega}: \varphi_{j}=x_{j}, 1 \leq j \leq n$. Therefore $y=\varphi(x)=x$ in $\bar{\Omega}$. This implies that $\tilde{\Omega}=\Omega$. Also $\varphi_{j}(x)=x_{j}$ implies (cf. (2.21)) that $\tilde{v}^{j}(x)=v^{j}(x), 1 \leq j \leq n$.

Therefore $\tilde{w}_{j}(x)=w_{j}(x), 1 \leq j \leq n$.

\section{Optical Black Holes}

In this section we explore the situation when the condition (1.3) is not satisfied.

Denote by $S$ the surface in $\Omega$ given by the equation $\Delta(x)=0$, where

$$
\Delta(x)=\operatorname{det}\left[g^{j k}(x)\right]_{j, k=1}^{n} .
$$

We assume that $S$ is a smooth and closed surface. Denote by $\Omega_{\text {ext }}$ the exterior of $S$ and by $\Omega_{\text {int }}$ the interior of $S$. We assume that $\Delta>0$ in $\Omega_{\text {ext }} \cap \bar{\Omega}, \Delta<0$ in $\Omega_{\text {int }}$ near $S$. It follows from Proposition 1.1 that the equation of $S$ can be written in the form $g_{00}(x)=0$ and $g_{00}(x)>0$ in $\Omega_{e x t} \cap \bar{\Omega}, g_{00}<0$ in $\Omega_{\text {int }}$ near $g_{00}(x)=0$. We shall write often the equation of $S$ in the form $S(x)=0$ and we assume that $\frac{\partial S(x)}{\partial x}$ is an outward normal to the surface $S(x)=0$.

In the case of the Gordon metric the equation of $S$ has the form

$$
\left(n^{2}(x)-1\right) \sum_{j=1}^{n}\left(v^{j}(x)\right)^{2}-1=0
$$

or, equivalently,

$$
\sum_{j=1}^{n} w_{j}^{2}(x)-\frac{c^{2}}{n^{2}(x)}=0,
$$

and the domain $\Omega_{\text {int }}$ is given by the inequality

$$
\sum_{j=1}^{n} w_{j}^{2}(x)>\frac{c^{2}}{n^{2}(x)}
$$

near $S(x)=0$.

Suppose that $S(x)=0$ is a characteristic surface of Eq. (1.1), i.e.

$$
\sum_{j, k=1}^{n} g^{j k}(x) S_{x_{j}}(x) S_{x_{k}}(x)=0 \quad \text { when } \quad S(x)=0 .
$$


Let $x_{j}=x_{j}(s), \xi_{j}=\xi_{j}(s), 0 \leq j \leq n, s \geq 0$ be a null-bicharacteristic of (1.1), i.e.

$$
\begin{aligned}
& \frac{d x_{j}}{d s}=2 \sum_{k=0}^{n} g^{j k}\left(x(s) \xi_{j}(s), x_{j}(0)=y_{j}, 0 \leq j \leq n,\right. \\
& \frac{d \xi_{p}}{d s}=-\sum_{j, k=0}^{n} g_{x_{p}}^{j k}(x(s)) \xi_{j}(s) \xi_{k}(s), \quad \xi_{p}(0)=\eta_{p}, \quad 0 \leq p \leq n,
\end{aligned}
$$

where $x(s)=\left(x_{1}(s), \ldots, x_{n}(s)\right), \eta_{0}=0, \eta \neq 0$. The null-bicharacteristic means that

$$
\sum_{j, k=0}^{n} g^{j k}(x(s)) \xi_{j}(s) \xi_{k}(s)=0 .
$$

Note that if

$$
\sum_{j, k=0}^{n} g^{j k}(y) \eta_{j} \eta_{k}=0
$$

then (3.6) holds for all $s \in \mathbf{R}$.

The bicharacteristic (null-bicharacteristic) is a curve in $T_{0}\left(\mathbf{R}^{n+1}\right)=\mathbf{R}^{n+1} \times\left(\mathbf{R}^{n+1} \backslash\right.$ $\{0\})$ and its projection on $\mathbf{R}^{n+1}$ is called a geodesic (null-geodesic). It is easy to show that the null-geodesic satisfies the equation:

$$
\sum_{j, k=0}^{n} g_{j k}(x(s)) \frac{d x_{j}}{d s} \frac{d x_{k}}{d s}=0 .
$$

Denote by $G$ the matrix $\left[g^{j k}(x)\right]_{j, k=0}^{n}$. Then $G^{-1}=\left[g_{j k}(x)\right]_{j, k=0}^{n}$. It follows from (3.4) that $\frac{d \vec{x}}{d s}=2 G \vec{\xi}(s)$, where $\vec{x}(s)=\left(x_{0}(s), x(s)\right), \vec{\xi}=\left(\xi_{0}, \xi\right)$. Then $\vec{\xi}=\frac{1}{2} G^{-1} \frac{d \vec{x}}{d s}$ and (3.6) implies

$$
0=(G \vec{\xi}, \vec{\xi})=\frac{1}{4}\left(G G^{-1} \frac{d \vec{x}}{d s}, G^{-1} \frac{d \vec{x}}{d s}\right)=\frac{1}{4}\left(G^{-1} \frac{d \vec{x}}{d s}, \frac{d \vec{x}}{d s}\right),
$$

that is equivalent to (3.8). $\mathbf{R}^{n+1}$ :

Fix an arbitrary point $y$ on $S(x)=0$. Denote by $K^{+}(y)$ the following half-cone in

$$
K^{+}(y)=\left\{\vec{\xi}=\left(\xi_{0}, \xi\right) \in \mathbf{R}^{n+1}:(G(y) \vec{\xi}, \vec{\xi}) \geq 0, \xi_{0} \geq 0\right\} .
$$

Let $K_{+}(y)$ be the dual half-cone in $\mathbf{R}^{n+1}$ :

$$
K_{+}(y)=\left\{\overrightarrow{\dot{x}}=\left(\dot{x}_{0}, \dot{x}\right) \in \mathbf{R}^{n+1}:\left(G^{-1} \overrightarrow{\dot{x}}, \overrightarrow{\dot{x}}\right) \geq 0, \dot{x}_{0}>0\right\} .
$$

Note that

$$
(\overrightarrow{\dot{x}}, \vec{\xi}) \geq 0 \text { for any } \overrightarrow{\dot{x}} \in K_{+}(y) \text { and any } \vec{\xi} \in K^{+}(y) \text {. }
$$

Consider the null-bicharacteristic $(\vec{x}(s), \vec{\xi}(s))$ (cf. (3.4), (3.5), (3.6)) with the following initial conditions:

$$
x_{0}(0)=y_{0}, \quad x(0)=y, \quad \xi_{0}(0)=0, \quad \xi(0)=S_{x}(y),
$$


where $S(y)=0$. We have (cf. (3.4)):

$$
\frac{d x_{0}(0)}{d s}=2 \sum_{j=1}^{n} g^{0 j}(y) S_{x_{j}}(y) .
$$

Proposition 3.1. Let $(0, b(y))$ be a characteristic direction, i.e.

$$
\sum_{j=1}^{n} g^{j k}(y) b_{j}(y) b_{k}(y)=0 .
$$

Suppose

$$
\sum_{j=1}^{n} g^{j 0}(y) b_{j}(y)>0 .
$$

Then $(0, b(y)) \in K^{+}(y)$ and the half-cone $K_{+}(y)$ is contained in the half-space $\left\{\left(\alpha_{0}, \alpha_{1}, \ldots, \alpha_{n}\right): \sum_{j=1}^{n} \alpha_{j} b_{j}(y) \geq 0\right\}$.

Proof. Let $\varepsilon>0$ be small. Then $\left(\varepsilon, b_{1}(y), b_{2}(y), \ldots, b_{n}(y)\right) \in K^{+}(y)$ since $g^{00}(y) \varepsilon^{2}+$ $2 \varepsilon \sum_{j=1}^{n} g^{j 0}(y) b_{j}(y)>0$ (cf. (3.10)). For any $\left(\dot{x}_{0}, \dot{x}\right) \in K_{+}(y)$ we have (cf. (3.12)):

$$
\dot{x}_{0} \varepsilon+\sum_{j=1}^{n} \dot{x}_{j} b_{j}(y) \geq 0
$$

i.e. $K_{+}(y)$ is contained in the half-space $\varepsilon \alpha_{0}+\sum_{j=1}^{n} \alpha_{j} b_{j}(y) \geq 0$. Taking the limit when $\varepsilon \rightarrow 0$ we prove Proposition 3.1

It follows from the hyperbolicity of (1.1) and from (3.3) that the right hand side of (3.14) is not zero. In fact, the hyperbolicity implies that the equation $\sum_{j, k=0}^{n} g^{j k}(y) \xi_{j} \xi_{k}=$ 0 has two distinct real roots $\xi_{0}^{(1)}(\xi), \xi_{0}^{(2)}(\xi)$ for any $\xi \neq 0$. Taking $\xi=S_{x}(y)$ we get $g^{00}(y) \xi_{0}^{2}+2 \sum_{j=1}^{n} g^{0 i}(y) \xi_{0} S_{x_{j}}(y)=0$, i.e. $\xi_{0}^{(1)}=0, \xi_{0}^{(2)}=-2\left(g^{00}(y)\right)^{-1}$ $\sum_{j=1}^{n} g^{0 j}(y) S_{x_{j}}(y) \neq 0$.

We assumed that $S_{x}(x)$ is the outward normal to the $S(x)=0$.

Since $S(x)$ satisfies (3.3) when $S(x)=0$ we have that either

$$
\sum_{j=1}^{n} g^{0 j}(y) S_{x_{j}}(y)>0, \quad \forall y \quad \text { such that } S(y)=0,
$$

or

$$
\sum_{j=1}^{n} g^{0 j}(y) S_{x_{j}}(y)<0, \quad S(y)=0 .
$$

If (3.15) holds then by Proposition $3.1\left(0, S_{x}(y)\right) \in K^{+}(y), \forall y, S(y)=0$, and $K_{+}(y)$ is contained in the half-space $\left(0, S_{x}(y)\right) \cdot\left(\dot{x}_{0}, \dot{x}\right) \geq 0$.

Note that the forward domain of influence of the point $y, S(y)=0$, consists of all forward time-like rays and null-geodesics that have the direction $\left(\dot{x}_{0}, \dot{x}\right) \in K_{+}(y)$ at the 
point $y$. All directions of $K_{+}(y)$ except $(1,0, \ldots, 0)$ are pointed inside $\Omega_{\text {ext }} \times \mathbf{R}$. Therefore the forward domain of influence of the surface $\{S(x)=0\} \times \mathbf{R}$ does not intersect $\Omega_{i n t} \times \mathbf{R}$. In such case the surface $\{S(x)=0\} \times \mathbf{R}$ is called the boundary of a white hole. Note that the forward domain of influence of $\Omega_{\text {ext }} \times \mathbf{R}$ does intersect $\Omega_{\text {int }} \times \mathbf{R}$.

Consider now the case when (3.16) holds. Then (cf. Proposition 3.1) the dual halfcone $K_{+}(y)$ is contained in the half-space $\overrightarrow{\dot{x}} \cdot\left(0,-S_{x}(y)\right) \geq 0$ and all $\overrightarrow{\dot{x}} \in K_{+}(y)$ (except $(1,0, \ldots, 0))$ are pointed inside $\Omega_{i n t} \times \mathbf{R}$. Therefore the domain of influence of $\bar{\Omega}_{i n t} \times \mathbf{R}$ is contained in $\bar{\Omega}_{\text {int }} \times \mathbf{R}$.

The surface $\{S(x)=0\} \times \mathbf{R}$ is called the boundary of a black hole in this case.

We proved the following theorem:

Theorem 3.1. Let $S(x)=\operatorname{det}\left[g^{j k}\right]_{j, k=1}^{n}=0$ be a closed and smooth characteristic surface, $g_{00}(x)>0$ in $\Omega_{\text {ext }} \cap \bar{\Omega}, g_{00}(x)<0$ in $\Omega_{\text {int }}$ near $S(x)=0$. Let $\left(0, S_{x}(x)\right)$ be the outward normal to the surface $\{S(x)=0\} \times \mathbf{R}$. Then $\{S(x)=0\} \times \mathbf{R}$ forms a white hole if (3.15) holds, and a black hole if (3.16) holds.

The application of Theorem 3.1 to the Gordon equation yields the following result (cf. [V]):

Theorem 3.2. Let $S(x)=0$ be the surface $\sum_{j=1}^{n} w_{j}^{2}(x)=\frac{c^{2}}{n^{2}(x)}$ and let $\{S(x)=0\} \times \mathbf{R}$ be a characteristic surface for the Gordon equation. Then $\{S(x)=0\} \times \mathbf{R}$ forms a white hole if $w(x)=\left(w_{1}(x), \ldots, w_{n}(x)\right)$ is pointed inside $\Omega_{\text {ext }}$ when $S(x)=0$ and $\{S(x)=0\} \times \mathbf{R}$ forms a black hole if $w(x)$ is pointed inside $\Omega_{\text {int }}$ when $S(x)=0$.

Proof. Since $\{S(x)=0\} \times \mathbf{R}$ is a characteristic surface for the Gordon equation we have (cf. (2.8)):

$$
\left|S_{x}(x)\right|^{2}=\frac{\left(n^{2}-1\right)}{c^{2}\left(1-\frac{|w|^{2}}{c^{2}}\right)}\left(\sum_{j=1}^{n} w_{j}(x) S_{x_{j}}(x)\right)^{2} .
$$

Therefore

$$
\left(c^{2}-|w|^{2}\right)\left|S_{x}\right|^{2}=\left(n^{2}-1\right)\left(w(x) \cdot S_{x}(x)\right)^{2} .
$$

Since $|w|^{2}=\frac{c^{2}}{n^{2}(x)}$ when $S(x)=0$ we get $\left|S_{x}\right|^{2}|w|^{2}=\left(w \cdot S_{x}\right)^{2}$. This last equality holds iff $S_{x}(x)=\alpha(x) w(x)$, where $S(x)=0, \alpha(x) \neq 0$. Since $S_{x}(x)$ is an outward normal we have that $\alpha(x)>0$ if $w(x)$ is pointed outwardly, and $\alpha(x)<0$ if $w(x)$ is pointed inwardly. In the case of the Gordon equation $g^{0 j}(x)=\frac{\left(n^{2}-1\right)}{c\left(1-\frac{|w|^{2}}{c^{2}}\right)} w_{j}(x)$ (cf. (2.10)). We have $\sum_{j=1}^{n} g^{0 j}(x) S_{x_{j}}(x)=\frac{\left(n^{2}-1\right)}{c\left(1-\frac{|w|^{2}}{c^{2}}\right)} \alpha(x)|w|^{2}$. Therefore (3.15) holds when $w(x)$ is pointed into $\Omega_{\text {ext }}$ and (3.16) holds when $w(x)$ is pointed into $\Omega_{i n t}$. In the first case we have a white hole and in the second case we have a black hole.

We shall study now the impact of the existence of the black or white hole on the uniqueness of the inverse problem.

Consider the case of a white hole. Then the domain of the dependence (i.e. the backward domain of influence) of any point $\left(y_{0}, y\right) \in \bar{\Omega}_{i n t} \times(-\infty,+\infty)$ is contained in $\bar{\Omega}_{i n t} \times(-\infty,+\infty)$. If $u\left(x_{0}, x\right)$ is the solution of the initial-boundary value problem 
(1.1), (1.5), (1.6) we get that $u=0$ in $\bar{\Omega}_{i n t} \times(-\infty,+\infty)$ by the uniqueness of the Cauchy problem. Therefore the change of the coefficients of $(1.1)$ in $\Omega_{\text {int }} \times(-\infty,+\infty)$ does not change the solution of the initial-boundary value problem (1.1), (1.5), (1.6) in $\left(\bar{\Omega} \cap \Omega_{\text {ext }}\right) \times(-\infty,+\infty)$ and it does not change the Cauchy data on $\partial \Omega_{0} \times(-\infty,+\infty)$. Therefore the boundary data on $\partial \Omega_{0} \times(-\infty,+\infty)$ are not able to determine the coefficients of (1.1), (modulo (1.10), (1.11)) in $\Omega_{\text {int }}$, i.e. we have a nonuniqueness.

Consider now the case of a black hole. Then the domain of influence of each point $\left(y_{0}, y\right) \in \bar{\Omega}_{i n t} \times \mathbf{R}$ is contained in $\bar{\Omega}_{i n t} \times \mathbf{R}$ and the domain of dependence of each point $\left(y_{0}, y\right) \in \bar{\Omega}_{\text {ext }} \times \mathbf{R}$ is contained in $\bar{\Omega}_{\text {ext }} \times \mathbf{R}$.

Consider the initial-boundary value problem (1.5), (1.6) for two hyperbolic equations $L^{(i)} u^{(i)}=0$ in $\Omega \times(-\infty,+\infty)$ whose coefficients differ in $\Omega_{\text {int }}$. Since the domain of dependence of any $\left(y_{0}, y\right) \in \bar{\Omega}_{\text {ext }} \times \mathbf{R}$ is contained in $\Omega_{\text {ext }} \times \mathbf{R}$ the solutions $u^{(i)}, i=1,2$, of the initial-boundary value problem (1.5), (1.6) are equal in $\bar{\Omega}_{\text {ext }} \times(-\infty,+\infty)$. Therefore the $\Lambda^{(1)}$ and $\Lambda^{(2)}$ are equal on $\partial \Omega_{0} \times(-\infty,+\infty)$, i.e. the boundary measurements are equal despite the fact that the coefficients of $L^{(1)}$ and $L^{(2)}$ differ in $\Omega_{i n t}$. Therefore we again have a non-uniqueness of the solution of the inverse problem.

Change the formulation of the initial-boundary value problem allowing nonzero initial condition in $\Omega_{i n t} \times \mathbf{R}$ or allowing the nonzero right hand of (1.1) with support in $\bar{\Omega}_{i n t} \times \mathbf{R}$. Then $u\left(x_{0}, x\right)$ will be nonzero in $\Omega_{\text {int }} \times(-\infty,+\infty)$ but this will not effect the solution in $\Omega_{\text {ext }} \times \mathbf{R}$.

\section{Black Holes Inside the Ergosphere}

Let $S(x)=0, \Omega_{i n t}, \Omega_{e x t}$ be the same as in $\S 3$. Borrowing the terminology from general relativity we shall call $S(x)=0$ the ergosphere and $\Omega_{e}=\Omega_{\text {int }} \cap\{\Delta(x)<0\} \cap \Omega$ is an ergoregion (cf. [V]). We assume that $\Delta(x)<0$ in $\bar{\Omega}_{e} \backslash S$.

If $S=\{x: S(x)=0\}$ is a characteristic surface then $\left(\Omega_{\text {int }} \cap \Omega\right) \times \mathbf{R}$ is either a black or white hole (cf. Theorem 3.1). Now consider the case when $S$ is not characteristic at any point $y \in S$, i.e.

$$
\sum_{j, k=1}^{n} g^{j k}(y) v_{j}(y) v_{k}(y) \neq 0, \quad \forall y \in S,
$$

where $v(y)$ is the outward normal to $S$. Note that the quadratic form $\sum_{j, k=1}^{n} g^{j k}(x) \xi_{j} \xi_{k}$ has the signature $(1,-1, \ldots,-1)$ when $x \in \Omega_{e}$. The question is whether there exists a black or white hole inside $\Omega_{e} \times \mathbf{R}$. Consider the case $n=2$, i.e. the case of two space dimensions. In this case there are two families of characteristic curves $S^{ \pm}=$const:

$$
\sum_{j, k=1}^{2} g^{j k}(x) S_{x_{j}}^{ \pm} S_{x_{k}}^{ \pm}=0
$$

in a neighborhood of any point of $\bar{\Omega}_{e}$. Equation (4.2) can be factored and we get in the region $\Omega_{e}^{(1)}=\left\{x \in \Omega_{e}: g^{22}(x) \neq 0\right\}$ :

$$
g^{22}(x) \frac{\partial S^{ \pm}(x)}{\partial x_{2}}+\left(g^{12}(x) \pm \sqrt{-\Delta(x)}\right) \frac{\partial S^{ \pm}}{\partial x_{1}}=0 .
$$

Note that $\Delta(x)=g^{11}(x) g^{22}(x)-\left(g^{12}(x)\right)^{2}<0$ in $\Omega_{e}$. 
We shall derive an equation for the characteristic curves $S^{ \pm}(x)=$ const that holds in a neighborhood of any point in $\bar{\Omega}_{e}$. Let $U_{1}$ be the set in $\bar{\Omega}_{e}$, where either $g^{22}(x) \neq$ 0 or $g^{12}(x)>0$. Denote by $f_{U_{1}}^{+}(x)$ a nonzero vector field $f_{U_{1}}^{+}(x)=\left(g^{12}(x)+\right.$ $\left.\sqrt{-\Delta(x)}, g^{22}(x)\right), x \in U_{1}$. Let $U_{2}$ be the set where either $g^{11}(x) \neq 0$ or $g^{12}(x)<0$ and denote by $f_{U_{2}}^{+}(x)$ the nonzero vector field $f_{U_{2}}^{+}(x)=\left(g^{11}(x), g^{12}(x)-\sqrt{-\Delta(x)}\right), x \in$ $U_{2}$. Let $U_{i}^{c}=\bar{\Omega}_{e} \backslash U_{i}, i=1,2$. Note that $U_{1}^{c} \cap U_{2}^{c}=\left\{x: g^{11}=g^{22}=g^{12}=0\right\}=\emptyset$, since the rank of $\left[g^{j k}\right]_{j, k=1}^{2}$ is at least 1 . Therefore $U_{1} \cup U_{2}=\bar{\Omega}_{e}$. Let $V_{i}, i=1,2$, be a small neighborhood of $U_{i}^{c}, i=1,2$, such that $\bar{V}_{1} \cap \bar{V}_{2}=\emptyset$. Denote $\tilde{U}_{i}=U_{i} \backslash V_{i}, i=$ 1, 2. Then $\tilde{U}_{1} \cup \tilde{U}_{2}=\Omega_{e}$. Note that

$$
\frac{g^{11}(x)}{g^{12}(x)+\sqrt{-\Delta}}=\frac{g^{12}-\sqrt{-\Delta}}{g^{22}(x)}=\lambda(x) \neq 0 \text { in } \tilde{U}_{1} \cap \tilde{U}_{2},
$$

since $g^{11} g^{22}=\left(g^{12}-\sqrt{-\Delta}\right)\left(g^{12}+\sqrt{-\Delta}\right)$.

Extend $\lambda(x)$ as a nonzero function from $\tilde{U}_{1} \cap \tilde{U}_{2}$ to $\tilde{U}_{2}$ such that $\lambda(x)$ is continuous in $\tilde{U}_{2}$ and smooth in $\tilde{U}_{2} \backslash S$, and define

$$
f^{+}(x)=f_{U_{1}}^{+}(x) \text { in } \tilde{U}_{1}, \quad f^{+}(x)=\lambda^{-1}(x) f_{U_{2}}^{+}(x) \text { in } \tilde{U}_{2} .
$$

Then $f^{+}$is a continuous nonzero vector field in $\bar{\Omega}_{e}$ that is smooth in $\Omega_{e} \backslash S$. Analogously let $f_{U_{3}}^{-}(x)=\left(g^{12}-\sqrt{-\Delta}, g^{22}\right), f_{U_{4}}^{-}(x)=\left(g^{11}, g^{12}+\sqrt{-\Delta}\right)$, where $U_{3}$ is the set where either $g^{22} \neq 0$ or $g^{12}<0$ and $U_{4}$ is the set where either $g^{11} \neq 0$ or $g^{12}>0$. Then $f_{U_{3}}^{-}(x)=\lambda_{1}(x) f_{U_{4}}^{-}(x)$ in $\tilde{U}_{3} \cap \tilde{U}_{4}$ and $\lambda_{1} \neq 0$ in $\tilde{U}_{3} \cap \tilde{U}_{4}$. Here $\tilde{U}_{3} \subset U_{3}, \tilde{U}_{4} \subset U_{4}$ are similar to $\tilde{U}_{i}, i=1,2$, in (4.4). Extending $\lambda_{1}(x)$ from $\tilde{U}_{3} \cap \tilde{U}_{4}$ to $\tilde{U}_{4}$ we get a vector field $f^{-}(x)$ in $\bar{\Omega}_{e}$ that is continuous in $\bar{\Omega}_{e}$ and smooth in $\bar{\Omega}_{e} \backslash S$.

We have that (4.2) is equivalent to

$$
f_{1}^{ \pm}(x) S_{x_{1}}^{ \pm}(x)+f_{2}^{ \pm}(x) S_{x_{2}}^{ \pm}(x)=0,
$$

where $f^{ \pm}(x)=\left(f_{1}^{ \pm}(x), f_{2}^{ \pm}(x)\right)$. Note that $f^{ \pm}(x) \neq(0,0)$ for any $x \in \bar{\Omega}_{e}$ and $f^{+}(x) \neq f^{-}(x), \forall x \in \Omega_{e} \backslash S$.

Since the rank of $\left[g^{j k}(y)\right]_{j, k=1}^{2}$ is 1 on $S$ there exists a smooth and nonzero $b(y)=$ $\left(b_{1}(y), b_{2}(y)\right)$ such that

$$
\sum_{k=1}^{2} g^{j k}(y) b_{k}(y)=0, \quad j=1,2, \quad y \in S .
$$

Since

$$
\sum_{j, k=1}^{2} g^{j k}(y) b_{j}(y) b_{k}(y)=0,
$$

we get from (4.1) that $b(y)$ is not co-linear with $v(y), \forall y \in S$. It follows from (4.5) with $S_{x}^{ \pm}(y)$ replaced by $b(y)$ that

$$
f_{1}^{ \pm}(y) b_{1}(y)+f_{2}^{ \pm}(y) b_{2}(y)=0, \quad \forall y \in S .
$$


Note that $f^{+}(y)=f^{-}(y)$ when $y \in S$. Therefore the condition (4.1) implies that $f^{ \pm}(y)$ is not tangential to $S$ for any $y \in S$. Therefore changing $f^{ \pm}(x)$ to $-f^{ \pm}(y)$ if needed we will assume that $f^{ \pm}(y)$ is pointed inside $\Omega_{e}$ for any $y \in S$.

Consider differential equations

$$
\frac{d \hat{x}^{ \pm}(\sigma)}{d \sigma}=f^{ \pm}\left(x^{ \pm}(\sigma)\right), \quad \hat{x}^{ \pm}(0)=y, \quad y \in S, \quad \sigma \geq 0 .
$$

Let $S^{ \pm}(x)=$ const be a characteristic curve. Then (4.5), (4.9) imply that $\frac{d}{d \sigma} S^{ \pm}\left(\hat{x}^{ \pm}(\sigma)\right)=$ 0 , i.e. $S^{ \pm}\left(\hat{x}^{ \pm}(\sigma)\right)=S^{ \pm}(y), \sigma \geq 0$. Therefore $x=\hat{x}^{ \pm}(\sigma)$ are the parametric equations of the characteristic curves $S^{ \pm}(x)=S^{ \pm}(y)$. It was shown in $\S 3$ that $\sum_{j=1}^{2} g^{0 j}(y) b_{j}(y) \neq$ 0 if $b(y)=\left(b_{1}, b_{2}\right)$ satisfies (4.7). Changing $b(y)$ to $-b(y)$ if needed we assume that

$$
\sum_{j=1}^{2} g^{0 j}(y) b_{j}(y)>0, \quad \forall y \in S .
$$

We shall denote by $S^{ \pm} \times \mathbf{R}$ the characteristic surfaces in $\Omega_{e} \times \mathbf{R}$, where $S^{ \pm}(x)=$ const and $x_{0} \in \mathbf{R}$. Let $\left(y_{0}, y^{ \pm}\right)$be any point of $S^{ \pm} \times \mathbf{R}$. Consider the null-bicharacteristic (3.4), (3.5) with initial conditions $x_{0}^{ \pm}(0)=y_{0}, x^{ \pm}(0)=y^{ \pm}, \xi^{ \pm}(0)=S_{x}^{ \pm}\left(y^{ \pm}\right), \xi_{0}^{ \pm}(0)=0$. Note that $x=x^{ \pm}(s), x_{0}^{ \pm}=x_{0}(s), s \geq 0$ is the corresponding null-geodesic. We shall show that $S^{ \pm}\left(x^{ \pm}(s)\right)=S^{ \pm}(y)$, i.e. this null-geodesic remains on the characteristic surface $S^{ \pm} \times \mathbf{R}$. We have

$$
\sum_{j, k=0}^{2} g^{j k}\left(x^{ \pm}(s)\right) \xi_{j}^{ \pm}(s) \xi_{k}^{ \pm}(s)=0
$$

since $\sum_{j, k=0}^{2} g^{j k} \eta_{j}^{ \pm} \eta_{k}^{ \pm}=0, \eta_{0}^{ \pm}=0, \eta^{ \pm}=S_{x}^{ \pm}\left(y^{ \pm}\right)$(cf. (3.6), (3.7)). Note that $\xi_{0}^{ \pm}(s)=\xi^{ \pm}(0)=0$ since $\left[g^{j k}(x)\right]_{j, k=0}^{2}$ is independent of $x_{0}\left(\mathrm{cf}\right.$. (3.5)). Since $S^{ \pm}(x)$ is a solution of the eiconal equation $\sum_{j, k=1}^{2} g^{j k}(x) S_{x_{j}}^{ \pm}(x) S_{x_{k}}^{ \pm}(x)=0$ in an open neighborhood of $y^{ \pm}$in $\Omega_{e}$, we have that (cf. $[\mathrm{CH}]$ )

$$
\xi^{ \pm}(s)=S_{x}^{ \pm}\left(x^{ \pm}(s)\right)
$$

Note that $\left(\frac{d x_{0}^{ \pm}}{d s}, \frac{d x^{ \pm}}{d s}\right)=2 G\left(0, \xi^{ \pm}(s)\right)$. Therefore

$$
\sum_{j=1}^{2} \frac{d x_{j}^{ \pm}}{d s} S_{x_{j}}^{ \pm}\left(x^{ \pm}(s)\right)=\sum_{j=1}^{2} \frac{d x_{j}^{ \pm}}{d s} \xi_{j}^{ \pm}(s)=2 \sum_{j, k=0}^{2} g^{j k}\left(x^{ \pm}(s)\right) \xi_{j}^{ \pm}(s) \xi_{k}^{ \pm}(s)=0,
$$

i.e. $\frac{d}{d s} S^{ \pm}\left(x^{ \pm}(s)\right)=0, \forall s$.

Therefore the projection of the null-bicharacteristic $x_{0}=x_{0}^{ \pm}(s), x=x^{ \pm}(s), x^{ \pm}(0)=$ $y^{ \pm}, \xi_{0}=0, \xi=\xi^{ \pm}(s)$ on the $\left(x_{1}, x_{2}\right)$-plane belongs to the curve $S^{ \pm}(x)=S^{ \pm}\left(y^{ \pm}\right)$.

Fix arbitrary $y \in S$. Denote by $\Pi^{+}(y)$ the intersection of the half-plane

$$
\xi_{1} b_{1}(y)+\xi_{2} b_{2}(y)>0
$$

with $\Omega_{e}$. Analogously let $\Pi^{-}(y)$ be the intersection of the half-plane

$$
\xi_{1} b_{1}(y)+\xi_{2} b_{2}(y)<0
$$


with $\Omega_{e}$. Let $x=\hat{x}^{ \pm}(\sigma)$ be the solution of (4.9), $\hat{x}^{ \pm}(0)=y, \sigma \geq 0$. Note that $f^{+}(y)=$ $f^{-}(y) \in \bar{\Pi}^{+}(y) \cap \bar{\Pi}^{-}(y)$. However for $\sigma>0$ and small, either $\hat{x}^{+}(\sigma)$ belongs to $\Pi^{+}(y)$ and $\hat{x}^{-}(\sigma)$ to $\Pi^{-}(y)$, or vice versa. For definiteness let $\hat{x}^{+}(\sigma) \in \Pi^{+}(y)$ for $0<\sigma<\varepsilon$ and $\hat{x}^{-}(\sigma)$ belongs to $\Pi^{-}(y)$ for $0<\sigma<\varepsilon$. Condition (4.1) and the continuity in $y$ imply $\hat{x}^{+}(\sigma) \in \Pi^{+}(y), \hat{x}^{-}(\sigma) \in \Pi^{-}(y), 0<\sigma<\varepsilon$, for all $y \in S$. Each $\hat{x}^{ \pm}(\sigma)$ is a parametric equation of the characteristic curve $S^{ \pm}(x)=S^{ \pm}(y)$, i.e.

$$
S^{ \pm}\left(\hat{x}^{ \pm}(\sigma)\right)=S^{ \pm}(y), \quad \forall \sigma \geq 0 .
$$

Note that the curve $S^{ \pm}(x)=S^{ \pm}(y)$ is contained in $\Pi^{ \pm}(y)$ when $x \neq y$ and close to $y$.

Consider now two null-bicharacteristics

$$
x_{0}=x_{0}^{+}(s), \quad x=x^{+}(s), \quad \xi_{0}=0, \quad \xi=\xi^{+}(s), s \geq 0,
$$

with initial conditions $x_{0}^{+}(0)=0, x^{+}(0)=y, \xi^{+}(0)=b$ and

$$
x_{0}=x_{0}^{-}(s), x=x^{-}(s), \quad \xi_{0}=0, \quad \xi=\xi^{-}(s), s \geq 0,
$$

with initial conditions $x_{0}^{-}(0)=0, x^{-}(0)=y, \xi^{-}(0)=-b$.

Note that

$$
\frac{d x_{0}^{+}(s)}{d s}=2 \sum_{j=1}^{2} g^{j 0}\left(x^{+}(s)\right) \xi_{j}^{+}(s)>0,
$$

since (4.10) holds. Also we have that

$$
\frac{d x_{0}^{-}(s)}{d s}<0, \quad s \geq 0
$$

since $\xi^{-}(0)=-b$. Therefore $x_{0}=x_{0}^{+}(s), x=x^{+}(s)$ is a forward null-geodesics since $x_{0}$ is increasing when $s$ is increasing and $x=x_{0}^{-}(s), x=x^{-}(s)$ is a backward null-geodesics.

It follows from (3.5) and (4.6) that

$$
\frac{d x_{j}^{ \pm}(0)}{d s}=2 \sum_{k=1}^{2} g^{j k}(y) b_{k}(y)=0, j=1,2 .
$$

However

$$
\frac{d x^{ \pm}(s)}{d s}=2 G\left(x^{ \pm}(s)\right)\left(0, \xi^{ \pm}(s)\right) \neq 0, \quad s>0,
$$

since $\Delta(x) \neq 0$ in $\Omega_{e} \backslash S$.

Since $(0, b(y)) \in K^{+}(y)$ (cf. (3.10)) the dual half-cone $K_{+}(y)$ is contained in $\bar{\Pi}^{+}(y)$. Therefore the projection $x=x^{+}(s), s \geq 0$, of the null-bicharacteristic (4.15) satisfies

$$
S^{+}\left(x^{+}(s)\right)=S^{+}(y), s \geq 0 .
$$

Analogously, the projection $x=x^{-}(s)$ of the null-bicharacteristic $\left(4.15^{\prime}\right)$ on $\left(x_{1}, x_{2}\right)$ plane satisfies

$$
S^{-}\left(x^{-}(s)\right)=S^{-}(y), s \geq 0 .
$$


Comparing (4.14) and (4.18), (4.18') we get that $x=x^{ \pm}(s), x=\hat{x}^{ \pm}(\sigma)$ are a different parametrization of the same curve, i.e.

$$
x^{ \pm}\left(s^{ \pm}(\sigma)\right)=\hat{x}^{ \pm}(\sigma), \quad \sigma \geq 0,
$$

where $s^{ \pm}(0)=0, \frac{d s^{ \pm}(\sigma)}{d \sigma}>0$ for $\sigma>0$.

Remark 4.1. Note that if $x=x_{0}(s), x=x(s), \xi_{0}=0, \xi=\xi(s)$, satisfies (3.4), (3.5), then $x=x_{0}(-s), x=x(-s), \xi_{0}=0, \xi=-\xi(-s)$ also satisfies (3.4), (3.5). Therefore changing $s \geq 0$ to $-s$ in $\left(4.15^{\prime}\right)$ and combining $(4.15)$ and $\left(4.15^{\prime}\right)$ we get a forward nullbicharacteristic $x=x_{0}(s), x=x(s), \xi_{0}=0, \xi=\xi(s)$, defined on $(-\infty,+\infty)$ with initial conditions $x_{0}(0)=0, x(0)=y, \xi(0)=b$. The projection of this bicharacteristic on the $\left(x_{1}, x_{2}\right)$-plane has a singularity (caustic) at $x=y$.

Let $\Omega_{e} \subset \Omega \cap \Omega_{\text {int }} \cap\{\Delta<0\}$. We assume that the boundary of $\Omega_{e}$ consists of $S=\partial \Omega_{\text {int }}$ and $S_{1}$. Assume that $\Delta<0$ on $S_{1}$ and $\Omega_{e}$ is diffeomorphic to an annulus domain in $\mathbf{R}^{2}$.

Let $y$ be an arbitrary point on $S_{1}$. Consider the forward cone of influence $K_{+}(y)$. Let $K_{+}^{(0)}(y)$ be the projection of this cone on the $\left(x_{1}, x_{2}\right)$-plane. We assume that

$$
N(y) \cdot \dot{x}>0, \quad \forall y \in S_{1}, \forall \dot{x} \in K_{+}^{(0)}(y),
$$

where $N(y)$ is the outward unit normal to $S_{1}$ and $\dot{x}=\left(\dot{x}_{1}, \dot{x}_{2}\right) \in \mathbf{R}^{2}$ is any vector in $K_{+}^{(0)}(y)$. In particular, $S_{1}$ is not a characteristic curve. Note that condition (4.20) is equivalent to the condition that

$$
N(y) \cdot \frac{d x^{ \pm}\left(s_{1}^{ \pm}\right)}{d s}>0, \quad x^{ \pm}\left(s_{1}^{ \pm}\right)=y, \forall y \in S_{1},
$$

where $x^{ \pm}(s)$ is the projection on the $\left(x_{1}, x_{2}\right)$-plane of two forward null-bicharacteristics such that $x=x^{ \pm}(s)$ are parametric equations of two characteristics of the form (4.2) passing through $y \in S_{1}$. Note that $\frac{d x_{0}^{ \pm}\left(s_{1}^{ \pm}\right)}{d s}>0$ when $x^{ \pm}\left(s_{1}^{ \pm}\right) \in S_{1}$.

We shall say that conditions $\left(4.20^{\prime}\right)$ or $\left(4.21^{\prime}\right)$ are satisfied if $N(y) \cdot \dot{x}<0$ in (4.20) or $N(y) \cdot \frac{d x^{ \pm}(s)}{d s}<0$ in $(4.21)$.

Theorem 4.1. Suppose $\partial \Omega_{e}=S_{0} \cup S_{1}$, where $\Delta(x)=0$ on $S, \Delta(x)<0$ on $\bar{\Omega}_{e} \backslash S$. Suppose (4.1) holds on S. Suppose also that either (4.20) or (4.20') is satisfied on $S_{1}$. Then there exists a Jordan curve $S_{0}(x)=0$ between $S$ and $S_{1}$ such that $S_{0} \times \mathbf{R}$ is a characteristic surface, i.e. $S_{0} \times \mathbf{R}$ is a boundary of either a black or a white hole.

Proof. It was shown already that condition (4.1) implies the existence of two null-bicharacteristics $x_{0}=x_{0}^{ \pm}(s), x=x^{ \pm}(s), \xi_{0}^{ \pm}=0, \xi=\xi^{ \pm}(s), s \geq 0$, such that $x^{ \pm}(0)=$ $y \in S$ and $x^{ \pm}(s)$ after reparametrization $s=s^{ \pm}(\sigma), s^{ \pm}(0)=0, \frac{d s^{ \pm}(\sigma)}{d \sigma}>0, \sigma>0$, coincide with the solution $x=\hat{x}^{ \pm}(\sigma)$ of the differential equation (4.9): $x^{ \pm}(s(\sigma))=$ $\hat{x}^{ \pm}(\sigma), \sigma \geq 0$. Moreover $\frac{d x_{0}^{-}(s)}{d s}<0$, i.e. $x_{0}$ decreases when $\sigma$ (or $s$ ) increases, and $\frac{d x_{0}^{+}(s)}{d s}>0$.

Suppose condition (4.20) is satisfied. We shall show that there is no solution $x=$ $\hat{x}^{-}(\sigma)$ of

$$
\frac{d \hat{x}^{-}(\sigma)}{d \sigma}=f^{-}(\hat{x}(\sigma)),
$$

$\hat{x}(0)=y \in S$ that reaches $S_{1}$, i.e. $\hat{x}^{-}\left(\sigma_{1}\right)=y^{(1)}$ where $y^{(1)} \in S_{1}, \sigma_{1}>0$. 
Suppose such $x=\hat{x}^{-}(\sigma)$ exists. When $\sigma>\sigma_{1}, \quad x=\hat{x}^{-}(\sigma)$ leaves $\Omega_{e}$, since $S_{1}$ is not characteristic. Note that for the null-bicharacteristic whose projection is $x=$ $x^{-}(s(\sigma))=\hat{x}^{-}(\sigma)$, the time variable $x_{0}=x_{0}^{-}(s)$ is decreasing when $\sigma$ is increasing. Therefore $x=\hat{x}^{-}(\sigma)$ leaves $\Omega_{e}$ when $x_{0}$ is decreasing. From the other side the condition (4.20) implies that the projection of all null bi-characteristics passing through $y^{(1)} \in S_{1}$ leave $\Omega_{e}$ when $x_{0}$ increases. This contradiction proves that the limit set of the trajectory $x=\hat{x}^{-}(\sigma)$ is contained inside $\Omega_{e}$. By the Poincaré-Bendixson theorem (cf. $[\mathrm{H}]$ ) there exists a limit cycle, i.e. a closed periodic solution $x=z^{-}(\sigma)$ of (4.22) which has no points of self-intersection. Let $S_{0}(x)=0$ be the equation of this orbit. Then $S_{0} \times \mathbf{R}$ is a characteristic surface where $S_{0}=\left\{x: S_{0}(x)=0\right\}$. Other solutions of (4.22) are spiraled around $S_{0}$ when $x_{0} \rightarrow-\infty$.

Now we shall assume that the condition $\left(4.20^{\prime}\right)\left(\right.$ or $\left.\left(4.21^{\prime}\right)\right)$ is satisfied. These conditions mean that the projection on the $\left(x_{1}, x_{2}\right)$-plane of any null-bicharacteristic passing through $S_{1}$ enters $\Omega_{e}$ when $x_{0}$ increases. From the other side, consider the solution of

$$
\frac{d \hat{x}^{+}(\sigma)}{d \sigma}=f^{+}\left(\hat{x}^{+}(\sigma)\right), \quad \sigma \geq 0
$$

that starts on $S: \hat{x}(0)=y \in S$. We claim that this solution can not reach $S_{1}$. In fact if $x=\hat{x}^{+}(\sigma)$ reaches $S_{1}$ it will leave $S_{1}$ when $\sigma>\sigma_{1}$. We have $\frac{d x_{0}^{+}(0)}{d s}>0$ for the nullbicharacteristics whose projection on the $\left(x_{1}, x_{2}\right)$-plane is $x=\hat{x}^{+}(\sigma)$ after the change of the parameter $s=s^{+}(\sigma)$. Therefore $x=x^{+}(s)$ leaves $\Omega_{e}$ when $x_{0}$ increases. This contradiction shows that the limit set of $x=x^{+}(\sigma)$ is contained inside $\Omega_{e}$.

Again by the Poincaré-Bendixson theorem there exists a closed characteristic curve $S_{0}(x)=0$ without points of self-intersection. All other solutions of (4.22) spiral around $S_{0}$ when $x_{0} \rightarrow+\infty$.

Remark 4.2. Without additional assumptions it is impossible to specify whether $S_{0} \times \mathbf{R}$ is a boundary of a white or a black hole.

For example, assume that the condition (4.20) is satisfied. Then $S_{0}$ is a limit cycle for the equation $\frac{d \hat{x}^{-}(\sigma)}{d \sigma}=f^{-}\left(\hat{x}^{-}(\sigma)\right)$. Since $f^{-}(x) \neq f^{+}(x)$ for all $x \in \Omega_{e}$ we have that $f^{+}(x), x \in S_{0}$ is pointed either into the interior of $S_{0}$ for all $x \in S_{0}$ or into the exterior of $S_{0}$. In the first case $S_{0} \times \mathbf{R}$ forms a black hole and in the second case $S_{0} \times \mathbf{R}$ forms a white hole. In the second case we can apply the Poincaré-Bendixson theorem to Eq. (4.23) in the annulus domain between $S$ and $S_{0}$. Then there exists a limit cycle $S_{2}$ for the equation $\frac{d \hat{x}^{+}(\sigma)}{d \sigma}=f^{+}(\hat{x}(\sigma))$ between $S$ and $S_{1}$ and $S_{2} \times \mathbf{R}$ that is the boundary of a white hole. Also there exists a white or black hole formed by $S_{3} \times \mathbf{R}$, where $S_{3}(x)=0$ is a limit cycle for (4.23) between $S_{0}$ and $S_{1}$. Analogously if condition $\left(4.20^{\prime}\right)$ is satisfied then the limit cycle $S_{0}$ for Eq. (4.23) forms a white hole if $f^{-}(x)$ restricted to $S_{0}$ is pointed in the exterior of $S_{0}$ and there are two additional black or white holes if $\left.f^{-}(x)\right|_{S_{0}}$ is pointed into the interior of $S_{0}$.

Remark 4.3. The black or white holes described in Theorem 4.1 are stable in the sense that if we perturb slightly $\left[g^{j k}(x)\right]_{j, k=0}^{2}$ then assumptions of Theorem 4.1 remain valid and therefore the limit cycle solution will still exist. This is in contrast with black or white holes obtained by Theorem 3.1: when we perturb $\left[g^{j k}\right]_{j, k=0}^{3}$ then the surface $S=\{\Delta(x)=0\}$ may cease to be characteristic and the black or white hole at $S \times \mathbf{R}$ disappears. 
Now we shall formulate the application of Theorem 4.1 to the case of the Gordon equation.

Theorem 4.2. Let $\partial \Omega_{e}=S \cup S_{1}$. Assume that $|w(x)|^{2}=\frac{c^{2}}{n^{2}(x)}$ on $S$ and $|w(x)|^{2}>\frac{c^{2}}{n^{2}(x)}$ in $\bar{\Omega}_{e} \backslash S$. Suppose $w(y)$ is not co-linear with $v(y)$ on $S$, where $v(y)$ is the outward unit normal to $S$. Suppose that either

$$
\left(n^{2}(x)-1\right)^{\frac{1}{2}}(v(x) \cdot N)>1 \text { on } S_{1},
$$

or

$$
\left(n^{2}(x)-1\right)^{\frac{1}{2}}(v(x) \cdot N)<-1 \text { on } S_{1},
$$

where $v(x)=\left(1-\frac{|w(x)|^{2}}{c^{2}}\right)^{-\frac{1}{2}} \frac{w(x)}{c}, N(x)$ is the outward unit normal to $S_{1}$.

Then there exists a Jordan curve $S_{0}(x)=0$ between $S$ and $S_{1}$ such that $S_{0} \times \mathbf{R}$ is a characteristic surface, i.e. $S_{0} \times \mathbf{R}$ is the boundary of either black or white hole.

Proof. Note that $\Delta(y)=0$ is equivalent to $|w|^{2}=\frac{c^{2}}{n^{2}(y)}, y \in S$ in the case of the Gordon equation and $\Delta(x)<0$ implies $|w|^{2}>\frac{c^{2}}{n^{2}(y)}$ in $\bar{\Omega}_{e} \backslash S$.

Since $\Delta(y)=0$ there exists a smooth $b(y) \neq 0, y \in S$, such that $\sum_{k=1}^{2} g^{j k}(y) b_{k}(y)=$ $0, j=1,2$ (cf. (4.6)). For the Gordon equation we have

$$
-b_{j}(y)+\left(n^{2}-1\right)(v \cdot b) v^{j}=0, \quad j=1,2,
$$

i.e. $b(y)$ is co-linear with $w(y)$ on $S$. Therefore $b(y)$ is not co-linear with $v(y)$ since $w(y)$ is not co-linear, $\forall y \in S$. Note that condition (4.10) has the form $\left(n^{2}-1\right)(v \cdot b) v^{0}>0$, i.e. $b$ and $w$ has the same direction. We take $b=w$. Therefore $f^{+}(y)=f^{-}(y)$ is orthogonal to $w(y)$ and is not tangential to $S, \forall y \in S$.

Let $y^{(1)}$ be any point on $S_{1}$. Suppose (4.24) holds. Consider any null-bicharacteristic whose projection is passing through $y^{(1)}$. The equation

$$
\sum_{j, k=1}^{2} g^{j k}\left(y^{(1)}\right) \xi_{j}\left(y^{(1)}\right) \xi_{k}\left(y^{(1)}\right)=0
$$

has the following form for the Gordon equation:

$$
\left.-\left|\xi\left(y^{(1)}\right)\right|^{2}+\left(n^{2}\left(y^{(1)}\right)-1\right)\left(v\left(y^{(1)}\right) \cdot \xi\left(y^{(1}\right)\right)\right)^{2}=0 .
$$

Note that $\frac{d x_{0}(s)}{d s}=2\left(n^{2}-1\right) v^{0}(v \cdot \xi)>0$ implies that $(v(x(s)) \cdot \xi(s))>0$. It follows from (4.25) that

$$
v \cdot \xi=\frac{|\xi|}{\left(n^{2}-1\right)^{\frac{1}{2}}} .
$$

We have

$$
\begin{aligned}
\frac{d x_{j}}{d s}= & 2 \sum_{k=1}^{2} g^{j k} \xi_{k}=-2 \xi_{j}+2\left(n^{2}-1\right)(v \cdot \xi) v^{j}=-2 \xi_{j}+2\left(n^{2}-1\right)^{\frac{1}{2}}|\xi| v^{j} \\
& j=1,2
\end{aligned}
$$


Take the inner product of (4.27) with $N$, where $N$ is outward unit normal to $S_{1}$. We get

$$
\frac{d x}{d s} \cdot N=-2 \xi \cdot N+2|\xi|\left(n^{2}-1\right)^{\frac{1}{2}}(v \cdot N)
$$

Since $|\xi \cdot N| \leq|\xi|$ we get, using (4.24), that $\frac{d x}{d s} \cdot N>0$ on $S_{1}$. Note that $\frac{d x_{0}(s)}{d s}>0$. Therefore any null-bicharacteristic escapes $\Omega_{e}$ when $s>s_{0}$ (or when $x_{0}>x_{0}\left(s_{0}\right)$ ) where $x\left(s_{0}\right) \in S_{1}$.

Therefore by Theorem 4.1 we have a characteristic surface $S_{0} \times \mathbf{R}$. If $\left(n^{2}-1\right)^{\frac{1}{2}}$ $(v \cdot N)<-1$ then all null-bicharacteristics are entering $\Omega_{e}$ when $x_{0}$ increases. Therefore again we can apply Theorem 4.1 .

As in Remark 4.3 the black and white holes described in Theorem 4.2 are stable.

Now we shall refine Theorem 4.2 and specify when the characteristic surface $S_{0} \times \mathbf{R}$ is the boundary of a white hole and when it is the boundary of a black hole.

We shall say that the flow $w(x)=\left(w,(x), w_{2}(x)\right)$ is incoming if for any closed simple curve $\Gamma \subset \Omega_{e}$ containing $S_{1}$ there exists at least one point $y \in \Gamma$ such that $w(y) \cdot v(y)>0$, where $v(y)$ is a normal to $\Gamma$ pointed inside $\Gamma$. Analogously, the flow $w(x)$ is called outgoing in $\Omega_{e}$ if for any $\Gamma$ there exists $y \in \Gamma$ such that $w(y) \cdot v(y)<0$.

Theorem 4.3. Consider the Gordon equation in $\Omega_{e}$, where $\Omega_{e}$ is the same as in Theorems 4.1 and 4.2. Suppose $w(y)$ is not colinear with the normal to $S, \forall y \in S$, and (4.24) holds on $S_{1}$. Assume in addition that the flow $w(x)$ is incoming. Then there exists a black hole bounded by $S_{0} \times \mathbf{R}$, where $S_{0}$ is a Jordan curve between $S$ and $S_{1}$. If $\left(4.24^{\prime}\right)$ holds and the flow $w(x)$ is outgoing then there exists a white hole with the boundary $S_{0} \times \mathbf{R}$.

Proof. Suppose (4.24) holds. It was proven in Theorem 4.2 that there exists a characteristic surface $S_{0} \times \mathbf{R}$. Now using that the flow $w(x)$ is incoming we shall prove that $S_{0} \times \mathbf{R}$ is the boundary of a black hole.

Take any $y \in S_{0}$. Let $b_{1}(y)$ be a normal to $S_{0}$. Choose the direction of $b_{1}(y)$ such that $\sum_{j=1}^{2} g^{0 j}(y) b_{j 1}(y)=2\left(n^{2}(y)-1\right) v^{0}(y)\left(v(y) \cdot b_{1}(y)\right)>0$, i.e. $\left.b_{1}(y) \cdot w(y)>0\right)$. It follows from Proposition 3.1 that $K_{+}(y)$ (cf. (3.11)) is contained in the half-space $\left(\alpha_{0}, \alpha_{1}, \alpha_{2}\right) \cdot\left(0, b_{11}(y), b_{21}(y)\right) \geq 0$.

Since the flow $w(x)$ is incoming there exists $y_{0} \in S_{0}$ such that $w\left(y_{0}\right) \cdot v\left(y_{0}\right)>0$, where $v\left(y_{0}\right)$ is pointed inside $S_{0}$. Since $b_{1}\left(y_{0}\right) \cdot w\left(y_{0}\right)>0$ and $b_{1}\left(y_{0}\right)$ is co-linear with $v\left(y_{0}\right)$ we get that $b_{1}\left(y_{0}\right)$ is also pointed inside $S_{0}$. Since $b_{1}(y)$ is continuous in $y$ we have that $b_{1}(y)$ is pointed inside $S_{0}$ for all $y \in S_{0}$. Therefore $\left(\dot{x}_{0}, \dot{x}\right) \in K_{+}(y)$ are pointed inside $S_{0} \times \mathbf{R}$, i.e. $S_{0} \times \mathbf{R}$ is the boundary of a black hole.

Suppose that $\left(4.24^{\prime}\right)$ is satisfied and the flow $w(x)$ is outgoing. Let $b_{1}(y)$ be the same as above, i.e. $b_{1}(y)$ is a normal to $S_{0}$ and $b_{1}(y) \cdot w(y)>0$. Since the flow $w(x)$ is outgoing there exists $y_{0} \in S_{0}$ such that $w\left(y_{0}\right) \cdot v\left(y_{0}\right)<0$, where $v\left(y_{0}\right)$ is pointed inside $S_{0}$. Then $b_{1}\left(y_{0}\right)$ is a normal to $S_{0}$ that is pointed outside of $S_{0}$. Therefore $K_{+}\left(y_{0}\right)$ is pointed outside of $S_{0} \times \mathbf{R}$. The same is true for any $y \in S_{0}$ by the continuity. Therefore $S_{0} \times \mathbf{R}$ is the boundary of a white hole.

Example 4.1. (Acoustic black hole (cf. [V])) Consider a fluid flow in a vortex with the velocity field

$$
v=\left(v^{1}, v^{2}\right)=\frac{A}{r} \hat{r}+\frac{B}{r} \hat{\theta},
$$


where $r=|x|, \hat{r}=\left(\frac{x_{1}}{|x|}, \frac{x_{2}}{\mid x_{2}}\right), \theta=\left(-\frac{x_{2}}{|x|}, \frac{x_{1}}{|x|}\right), A$ and $B$ are constants. The inverse of the metric tensor in this case has the form

$$
\begin{aligned}
& g^{00}=\frac{1}{\rho c}, \quad g^{0 j}=g^{j 0}=\frac{1}{\rho c} v^{j}, 1 \leq j \leq 2, \\
& g^{j k}=\frac{1}{\rho c}\left(-c^{2} \delta_{i j}+v^{i} v^{j}\right), \quad 1 \leq j, k \leq 2,
\end{aligned}
$$

where $c$ is the sound speed, $\rho$ is the density.

It is convenient to use polar coordinates in (4.2). Assuming $c=1, \rho=1$ we have

$$
\left(\frac{A^{2}}{r^{2}}-1\right)\left(\frac{\partial S}{\partial r}\right)^{2}+\frac{2 A B}{r^{3}} \frac{\partial S}{\partial r} \frac{\partial S}{\partial \theta}+\left(\frac{B^{2}}{r^{4}}-\frac{1}{r^{2}}\right)\left(\frac{\partial S}{\partial \theta}\right)^{2}=0
$$

or (cf. (4.5))

$$
\left(\frac{A^{2}}{r^{2}}-1\right) \frac{\partial S^{ \pm}}{\partial r}+\left(\frac{A B}{r^{3}} \pm \sqrt{\frac{A^{2}+B^{2}}{r^{4}}-\frac{1}{r^{2}}}\right) \frac{\partial S^{ \pm}}{\partial \theta}
$$

for $r<\sqrt{A^{2}+B^{2}}$. The ergosphere, i.e. the curve where $\Delta=0$ has the form $A^{2}+$ $B^{2}=r^{2}$. We shall consider the domain $0<r<\sqrt{A^{2}+B^{2}}$ or any compact domain $\bar{\Omega}_{e}=\left\{r_{1} \leq r \leq r_{0}\right\}$ where $r_{0}=\sqrt{A^{2}+B^{2}}, r_{1}<|A|$. The condition (4.1) is satisfied when $B \neq 0$. The condition (4.20) is satisfied when $A>r_{1}$ and the condition (4.20') holds when $A<-r_{1}$.

Consider the case $A>0, B>0$. The system (4.9) has the following form in polar coordinates:

$$
\begin{aligned}
\frac{d r^{+}}{d s} & =A^{2}-r^{2}, \quad \frac{d \theta^{+}}{d s}=\frac{A B}{r}+\sqrt{A^{2}+B^{2}-r^{2}}, \\
\frac{d r^{-}(s)}{d s} & =-1, \quad \frac{d \theta^{-}(s)}{d s}=\frac{1-\frac{B^{2}}{r^{2}}}{\frac{A B}{r}+\sqrt{A^{2}+B^{2}-r^{2}}},
\end{aligned}
$$

$r^{ \pm}(0)=r_{0}, \theta^{ \pm}(0)=\frac{y}{|y|},|y|=r_{0}, s \geq 0$. Note that $\frac{d x}{d s}=\frac{d r}{d s} \hat{r}+r \frac{d \theta}{d s} \hat{\theta}$. We wanted to ensure that the vector field in the right hand sides of (4.32), (4.33) is not zero. Since $\frac{A B}{r}-\sqrt{A^{2}+B^{2}-r^{2}}=0$ when $A=r, B>0$ we divided by $r^{2}-A^{2}$ in (4.33) and used that $\frac{A B}{r}-\sqrt{A^{2}+B^{2}-r^{2}}=\frac{\left(r^{2}-A^{2}\right)\left(1-\frac{B^{2}}{r^{2}}\right)}{\frac{A B}{r}+\sqrt{A^{2}+B^{2}-r^{2}}}$. It is clear that $r^{2}=A^{2}$ is the limit cycle for Eq. (4.32).

In the notations of Theorem 4.2 (cf. (4.10), (4.29)) we have $b(y)=v(y)=\frac{A}{r_{0}} \hat{r}+\frac{B}{r_{0}} \hat{\theta}$, where $|y|=r_{0}=\sqrt{A^{2}+B^{2}}$. We have, (cf. (4.12), (4.13)):

$$
\begin{aligned}
\frac{d x^{+}}{d s} \cdot b(y) & =\left(\frac{d r^{+}}{d s} \hat{r}+r_{0} \frac{d \hat{\theta}(s)}{d s} \hat{\theta}\right) \cdot\left(\frac{A}{r_{0}} \hat{r}+\frac{B}{r_{0}} \hat{\theta}\right) \\
& =-\frac{A B^{2}}{r_{0}}+\frac{A B^{2}}{r_{0}}+B \sqrt{r_{0}^{2}-r^{2}(s)}+O\left(r_{0}-r(s)\right)>0 \text { for } 0 \leq s \leq \varepsilon .
\end{aligned}
$$


Analogously $\frac{d x^{-}(s)}{d s} \cdot b(y)<0$ for $0<s<\varepsilon, \varepsilon$ is small. Note that $r=r^{+}(s)$, $\theta=\theta^{+}(s)$ is the projection on the $\left(x_{1}, x_{2}\right)$-plane (after a reparametrization) of the null-bicharacteristic with initial conditions $x^{+}(0)=y,|y|=r_{0}, x_{0}^{+}(0)=0, \xi_{0}^{+}(0)=0, \xi(0)=$ $b(y)$ and $\frac{d x_{0}^{+}(s)}{d s}>0, \forall s \geq 0$.

When $x_{0} \rightarrow+\infty, \quad r=r^{+}(s), \theta=\theta^{+}(s)$ spirals toward the limit cycle $r=A$. Also $r=r^{-}(s), \theta=\theta^{-}(s)$ is the projection (after a reparametrization) of the nullbicharacteristic with initial conditions $x_{0}^{-}(0)=0, x^{-}(0)=y, \xi_{0}^{-}=0, \xi(0)=-b(y)$ and $\frac{d x_{0}^{-}(s)}{d s}<0$. Note that $r=r^{-}(s), \theta=\theta^{-}(s)$ reaches $r=r_{1}$ when $x_{0} \rightarrow-\infty$. Therefore $r=A$ is the boundary of a white hole.

When $A<-r_{1}, B>0$ we have, analogously to (4.32), (4.33):

$$
\begin{gathered}
\frac{d r^{+}}{d s}=-1, \quad \frac{d \theta^{+}}{d s}=\frac{1-\frac{B^{2}}{r^{2}}}{\frac{A B}{r}-\sqrt{A^{2}+B^{2}-r^{2}}}, \\
\frac{d r^{-}(s)}{d s}=A^{2}-r^{2}, \quad \frac{d \theta^{-}(s)}{d s}=\frac{A B}{r}-\sqrt{A^{2}+B^{2}-r^{2}},
\end{gathered}
$$

$r^{ \pm}(0)=r_{0}, \theta^{ \pm}(0)=\frac{y}{|y|},|y|=r_{0}$, and we modified (4.34) since $\frac{A B}{r}+\sqrt{A^{2}+B^{2}-r^{2}}$ $=0$ when $r=|A|$. Now $r=r^{-}(s), \theta=\theta^{-}(s)$ spirals towards $r=|A|$ when $x_{0} \rightarrow-\infty$ and $r=r^{+}(s), \theta=\theta^{+}(s)$ reaches $r=r_{1}$ when $x_{0} \rightarrow+\infty$. Therefore $r=|A|$ is the boundary of a black hole.

A similar result holds when $B<0$. The difference is that the spiraling of the solutions towards $r=|A|$ changes from the clockwise to the counter-clockwise or vice versa.

Example 4.2. Consider a generalization of Example 4.1 when

$$
v(x)=A(r) \hat{r}+B(r) \hat{\theta}
$$

where $r_{1} \leq r \leq r_{0}, A(r), B(r)$ are smooth, $A^{2}\left(r_{0}\right)+B^{2}\left(r_{0}\right)=1, A^{2}(r)+B^{2}(r)>1$ on $\left[r_{1}, r_{0}\right), B(r)>0$ on $\left[r_{1}, r_{0}\right], A(r)+1$ has simple zeros $\alpha_{1}, \ldots, \alpha_{m_{1}}$ on $\left(r_{1}, r_{0}\right), A(r)-1$ has simple zeros $\beta_{1}, \ldots, \beta_{m_{2}}$ on $\left(r_{1}, r_{0}\right), \beta_{k} \neq \alpha_{j}, \forall j, k,\left|A\left(r_{1}\right)\right|>1$. Equation (4.31) has the form

$$
\left(A^{2}(r)-1\right) S_{r}^{ \pm}+\left(A(r) B(r) \pm \sqrt{A^{2}+B^{2}-1}\right) S_{\theta}^{ \pm}=0 .
$$

Let

$$
\begin{aligned}
& \frac{d r^{+}(s)}{d s}=A(r)-1, \quad \frac{d \theta^{+}}{d s}=\frac{A(r) B(r)+\sqrt{A^{2}+B^{2}-1}}{A(r)+1}, \\
& \frac{d r^{-}(s)}{d s}=A(r)+1, \quad \frac{d \theta^{-}}{d s}=\frac{A(r) B(r)-\sqrt{A^{2}+B^{2}-1}}{A(r)-1} .
\end{aligned}
$$

Note that the right hand sides in (4.37), (4.38) are smooth since the singularities at $A(r)+1=0$ and $A(r)-1=0$ are removable.

It follows from (4.37), (4.38) that $\left\{|x|=\alpha_{j}\right\} \times \mathbf{R}, j=1, \ldots, m_{1}, \quad\left\{|x|=\beta_{k}\right\} \times$ $\mathbf{R}, k=1, \ldots, m_{2}$, are boundaries of black or white holes. In particular if $\alpha_{1}=$ $\min _{j, k}\left(\alpha_{j}, \beta_{k}\right)$ and $A\left(r_{1}\right)<-1$ then $r=\alpha_{1}$ is the boundaries of a black hole. If $\beta_{1}=\min _{j, k}\left(\alpha_{j}, \beta_{k}\right)$ and $A\left(r_{1}\right)>1$ then $r=\beta_{1}$ is the boundary of a white hole. 
Remark 4.4. (Axially symmetric metrics) Consider Eq. (1.1) in $\Omega \times \mathbf{R}$, where $\Omega$ is a three-dimensional domain. Let $(r, \theta, \varphi)$ be the spherical coordinates of $x=\left(x_{1}, x_{2}, x_{3}\right)$, i.e. $x_{1}=r \sin \theta \cos \varphi, x_{2}=r \sin \theta \sin \varphi, x_{3}=r \cos \theta$.

Suppose that $g^{j k}$ are independent of $\varphi: g^{j k}=g^{j k}(r, \theta)$. Consider the characteristic surface $S$ independent of $x_{0}$ and $\varphi$ :

$$
\sum_{j, k=1}^{3} g^{j k}(r, \theta) \frac{\partial S}{\partial x_{j}} \frac{\partial S}{\partial x_{k}}=0
$$

where

$$
\frac{\partial S}{\partial x_{k}}=\frac{\partial S}{\partial r} \frac{\partial r}{\partial x_{k}}+\frac{\partial S}{\partial \theta} \frac{\partial \theta}{\partial x_{k}}, \quad k=1,2,3 .
$$

Substituting (4.40) into (4.39) we get an equation:

$$
a^{11}(r, \theta)\left(\frac{\partial S}{\partial r}\right)^{2}+2 a^{12}(r, \theta) \frac{\partial S}{\partial r} \frac{\partial S}{\partial \theta}+a^{22}(r, \theta)\left(\frac{\partial S}{\partial \theta}\right)^{2}=0 .
$$

We assume that $a^{j k}(r, \theta)$ are also independent of $\varphi$. We consider (4.41) in the twodimensional domain $\omega$ such that $\delta_{1} \leq r \leq \delta_{2}, 0<\delta_{3}<\theta<\pi-\delta_{4}$ when $(r, \theta) \in \omega$. Imposing on $\omega$ and $a^{i j}(r, \theta), 1 \leq i, j \leq 2$, the conditions of Theorems 3.1 and 4.1, we shall prove the existence of black or white holes in $\Omega \times \mathbf{R}$ with the boundary of the form $S_{0} \times S^{1} \times \mathbf{R}$, where $\varphi \in S^{1}, x_{0} \in \mathbf{R}$ and $S_{0}$ is a closed Jordan curve in the $(r, \theta)$ plane.

Open Access This article is distributed under the terms of the Creative Commons Attribution Noncommercial License which permits any noncommercial use, distribution, and reproduction in any medium, provided the original author(s) and source are credited.

\section{References}

[B] Belishev, M.: Recent progress in the boundary control method. Inverse Problems 23(5), R1R67 (2007)

[CH] Courant, R., Hilbert, D.: Methods of Mathematical Physics. Vol. II, New York, London: WileyInterscience, 1962

[E1] Eskin, G.: Optical aharonov-bohm effect: inverse hyperbolic problem approach. Commun. Math. Phys. 284, 317-343 (2008)

[E2] Eskin, G.: A new approach to the hyperbolic inverse problems II: global step. Inverse Problems 23, 2343-2356 (2007)

[G] Gordon, W.: Ann. Phys. (Leipzig) 72, 421 (1923)

[H] Hartman, F.: Ordinary differential equations. New York: J.Wiley \& Son, 1964

[KKL] Katchalov, A., Kurylev, Y., Lassas, M.: Inverse boundary spectral problems. Boca Baton: Chapman\&Hall, 2001

[LP] Leonhardt, V., Piwnicki, P.: Phys. Rev. A60, 4301 (1999)

[NVV] Novello, M., Visser, M., Volovik, G.: (editors): Artificial black holes. Singapore: World Scientific, 2002

[U] Unruh, W.: Phys. Rv. Lett. 46, 1351 (1981)

[V] Visser, M.: Acoustic black holes, horizons, ergospheres and hawking radiation. Class. Quant. Grav. 15(6), 1767-1791 (1998)

Communicated by P. Constantin 